\title{
Growth of silver on zinc oxide via lattice and off-lattice adaptive kinetic Monte Carlo
}

\author{
Adam L. Lloyd ${ }^{\text {a) }}$ and Roger Smith \\ Department of Mathematical Sciences, Loughborough University, Loughborough LE11 3TU, U.K. \\ Steven D. Kenny ${ }^{\text {b) }}$ \\ Department of Materials, Loughborough University, Loughborough LE11 3TU, U.K.
}

(Received 2 October 2017; accepted 14 December 2017)

\begin{abstract}
The growth of $\mathrm{Ag}$ on $\mathrm{ZnO}$ was modeled using a reactive force field potential and a combination of molecular dynamics and adaptive kinetic Monte Carlo (AKMC) simulations. An adaptive lattice-based AKMC model is described as a method of extending timescales and length scales that can be simulated. Reusing previously found transitions to reduce computational time is discussed for both the lattice and off-lattice AKMC approaches. With these methods, growth of over 1 monolayer's worth of $\mathrm{Ag}$ is simulated corresponding to a real deposition time of up to $0.1 \mathrm{~s}$. The results show that the deposited silver aggregates on the surface through mainly single atom moves with few concerted motions. Initially silver adatoms do not agglomerate and the energy barriers for silver dimers to form are larger than for them to break apart. The first layer of silver grows as a series of connected regions rather than forming well-defined centro-symmetric islands.
\end{abstract}

\section{INTRODUCTION}

Low-Emissivity (Low-E) coatings are used to prevent heat loss (or gain) through windows. ${ }^{1}$ These coatings are designed to keep the heat inside (or outside) a building. Ideal Low-E windows have to transmit visible light and prevent transmission of select wavelengths of infra red light whilst maintaining a neutral appearance. The principle structure of a Low-E coating is a reflective layer sandwiched between two dielectric layers. Typically a thin film of silver, grown by magnetron sputtering is used as the reflective layer, while zinc oxide is used as the dielectric layer ${ }^{2}$ (however other dielectric materials have been investigated $\left.{ }^{3}\right)$. It is this application of the $\mathrm{Ag}-\mathrm{ZnO}$ interface that motivates the work described in this article.

The smoothness of the thin film is key to its performance as a Low-E coating. However, experimental results ${ }^{4}$ suggest that $\mathrm{Ag}$ grows in islands when deposited at low energies rather than uniform, flat layers. It has also been known for some time that the interface between $\mathrm{Ag}$ and $\mathrm{ZnO}$ is one of the weakest in a multilayer stack, and this has been the subject of previous investigations by a number of authors, see for example Refs. 5 and 6.

Contributing Editor: Danny Perez

${ }^{a)}$ Address all correspondence to this author.

e-mail: a.lloyd5@lboro.ac.uk

${ }^{b)}$ This author was an editor of this journal during the review and decision stage. For the JMR policy on review and publication of manuscripts authored by editors, please refer to http://www.mrs. org/editor-manuscripts/.

DOI: $10.1557 / j m r .2017 .482$
Until recently good models of atomistic growth processes were lacking in the literature. In many cases, the empirical potentials were inadequate to obtain a full description of the chemistry of the interaction between the arriving atoms, and the substrate and ab initio methods were too slow. Molecular dynamics (MD) could not be used on its own to model experiment since the technique could not access the time scales involved. Kinetic Monte Carlo (KMC) using pre-defined event lists might access longer times but could miss many important transitions.

Over the last few years, progress has been made to make growth simulations more realistic. First reactive force field potentials $^{7-9}$ have become accepted as a way to capture many of the effects missing from previous empirical potential descriptions and secondly adaptive KMC methods, introduced by Henkelman and Jónsson, ${ }^{10}$ can be used to predict unexpected transitions which would be missed by KMC using predefined event tables. ${ }^{11-13}$

Originally, a simplified model, using a combination of pair potentials for $\mathrm{Ag}-\mathrm{Zn}$ and $\mathrm{Ag}-\mathrm{O}$ interactions fitted to works of separation, ${ }^{6}$ was first considered for the simulation of $\mathrm{Ag}$ growth on $\mathrm{ZnO}$ surfaces. However, the model predicts that a single interstitial $\mathrm{Ag}$ atom is energetically favourable compared to a surface $\mathrm{Ag}$ adatom on the polar $\mathrm{ZnO}(000 \overline{1})$ surface by $0.75 \mathrm{eV}$ in contradiction to ab initio results. The ab initio calculations predict that the energetically favourable position for $\mathrm{Ag}$ is on the surface, the energy difference being $1 \mathrm{eV}$ compared to the sub-surface position. As a result, a more 
comprehensive many body potential function was developed for full thin film growth simulations. ${ }^{9}$

In a previous publication ${ }^{9}$ we reported on a new reactive force field (ReaxFF) potential, fit to a large set of ab initio data that captures many of the surface characteristics of $\mathrm{Ag}$ on $\mathrm{ZnO}$-including works of separation. This newer model predicts that the interstitial $\mathrm{Ag}$ is metastable compared to an $\mathrm{Ag}$ adatom in agreement to ab initio results. It is this potential that is used in the article for growth simulation combined with a multitime scale technique that uses MD to model the collision of an incoming $\mathrm{Ag}$ atom onto a $\mathrm{ZnO}$ surface followed by adaptive kinetic Monte Carlo (AKMC) to capture the surface rearrangements between impacts. Despite recent advances in the methodology used to speed up simulations, i.e., re-using previously discovered transitions and the implementation of superbasin methods, ${ }^{14-16}$ these calculations are very lengthy. Due to this, a lattice based adaptive KMC (Lat-AKMC) model was developed which can also harness some of the same advances in methodology. The Lat-AKMC method can deal with much larger systems and can access even longer time scales than the off-lattice approach.

\section{METHODOLOGY}

The two methodologies used to understand the growth process are described below.

\section{A. AKMC}

Time scales are extended using a combination of Molecular Dynamics (MD) and AKMC. The basic methodology has been described in previous work $^{12,13}$ and allows simulation of metallic systems over several seconds but for the more complex Ag- $\mathrm{ZnO}$ ReaxFF potential, the longest simulation time achieved was around $0.1 \mathrm{~s}$ of real deposition time on a 512 atom system. The AKMC approach has been used to identify many complex transition pathways and mechanisms for thin film growth. ${ }^{17}$

Initially a substrate of dimension $2.28 \times 0.81 \times$ $2.63 \mathrm{~nm}$ (with periodic boundary conditions in the $x$ and $z$ directions, parallel to the surface) was used which consisted of 4 double layers with $64 \mathrm{O}$ and $64 \mathrm{Zn}$ atoms in each layer. Despite a small system being simulated, the substrate's dimensions in the periodic directions are much larger than the $1 \mathrm{~nm}$ cut-off distance applied to the bond order potential function. A perfect O-terminated polar $(000 \overline{1})$ surface was first chosen but some surfaces containing $\mathrm{O}$ vacancies and steps were also considered. In all cases the substrate was initially relaxed using conjugate gradient minimisation until all forces acting on atoms were less than $0.025 \mathrm{eV} / \AA$. For all structures considered, relaxation resulted in no large changes in the surface configuration. The bottom layer of atoms was held fixed and the next two layers were connected to a Berendsen thermostat ${ }^{18}$ to keep the system temperature at $300 \mathrm{~K}$. The rate of deposition onto the surface was chosen as 12 monolayers per second $(\mathrm{mL} / \mathrm{s})$, which for our system size was equated to successive particles striking the surface in a time equivalent to a diffusion event occurring with an energy barrier of $0.6 \mathrm{eV}$.

In a magnetron, $\mathrm{Ag}$ atoms and ions strike the surface with an energy of a few $\mathrm{eV}$ at rate between 3 and $50 \mathrm{~mL} / \mathrm{s}$ dependent on a range of parameters such as power, current and argon pressure. ${ }^{19}$ As a simplified model of this process, each incoming $\mathrm{Ag}$ particle was assumed to be charge neutral and to strike the surface with an energy of $3 \mathrm{eV}$. Thus a randomly positioned $\mathrm{Ag}$ atom is given an energy level of $3 \mathrm{eV}$ and directed normally toward the surface. MD is then carried out for $10 \mathrm{ps}$, and afterward the system is relaxed using conjugate gradient minimisation. The parameters used in our model: namely the system temperature taken to be $300 \mathrm{~K}$, a deposition rate of $12 \mathrm{~mL} / \mathrm{s}$ and a deposition energy of $3 \mathrm{eV}$ were of particular interest to our experimental collaborators and are reasonable approximations of the conditions observed in their magnetron sputtering devices. Whilst in reality, the angle of incidence of the deposited Ag atoms may vary from normal, the exact distribution and range of angles are unknown. Despite this, whilst using low deposition energies, it would be expected that differences in incidence angles would have little effect on the initial growth mechanisms on a perfect $\mathrm{ZnO}$ surface but may have a larger influence once islands have formed.

After a deposition event, the AKMC procedure is then implemented. The AKMC procedure is slightly modified from that described in our previous work ${ }^{12}$ in that the open ended saddle point searches are conducted, not for the entire system but in neighbourhoods (typically all atoms within 6th nearest neighbor distance) of atoms that are not fully co-ordinated compared to typical bulk atoms or atoms in a perfect surface. Such a region will be termed a 'defect volume'. A typical defect volume used in the simulated growth of $\mathrm{Ag}$ on $\mathrm{ZnO}$ contains 40-50 moving atoms whilst the rest of the system is fixed and used for force calculations. In larger systems, subsystems containing $100 \mathrm{~s}$ of atoms can be used to calculate forces more efficiently within a defect volume; however, this technique was not used for the small $\mathrm{Ag} / \mathrm{ZnO}$ system considered in this work. For each defect volume, a graph is constructed based on the connectivity of atoms within the volume and given a canonical label via the topological software NAUTY,${ }^{20}$ this label is referred to as the 'graph key'. This is then used to store a database of atom positions and transitions corresponding to the defect volume. Overlapping defect volumes are combined to produce a larger defect volume. There are two main benefits of combining nearby defect volumes in this way. One is to eliminate duplicate transitions that may be found involving shared atoms between defect volumes. The other benefit is that combined volumes may allow transitions consisting of large concerted motions to be found via saddle point searches. 
For this system, saddle searches were conducted on each defect volume using the relaxation and translation (RAT) method, ${ }^{21}$ a modification of the activationrelaxation technique. ${ }^{22}$ This method was used ahead of other saddle searching techniques, such as the Dimer method, ${ }^{23}$ as it more consistently produced a large set of unique transition pathways for this system.

There are also many tricks used to speed up the searches such as re-use of previously found transitions. If a newly constructed defect volume is topologically equivalent to a previously found volume (i.e., has the same graph key), the stored transitions can then be utilised. The initial and final states of each transition from the database are transformed to best fit the local environment. A transformation between a stored defect volume, $\mathrm{DV}_{i}$, and newly found defect volume, $\mathrm{DV}_{j}$, is done by first calculating atomistic positions relative to the center of mass ${ }^{24}$ of each defect volume. Then an isomorphism is calculated such that each atom in volume $\mathrm{DV}_{i}$ has a corresponding atom in $\mathrm{DV}_{j}$ via NAUTY. An optimal transformation matrix is calculated as the best solution of an overdetermined linear system of equations using the method of least squares. The transformation is then applied to the stored volume and stored transitions to approximate the pathways of transitions for the current volume. Transition pathways are then refined to fit the local environment via the nudged elastic band method $(\mathrm{NEB})^{25}$ reducing the time spent conducting a full set of searches for new transition pathways. Similar methods have been described in the kinetic-ART methodology. ${ }^{16,26}$ However we use our own AKMC code rather than the one described in Ref. 16.

Once a transition or new deposition has been chosen via the Monte Carlo process, the system is relaxed, the clock is advanced and the procedure is repeated. The transition frequencies $v$ are calculated using the Arrhenius method where $v=v_{0} \exp \left(-E_{\mathrm{B}} / k_{\mathrm{B}} T\right) ; E_{\mathrm{B}}$ is the energy barrier, $k_{\mathrm{B}}$ is Boltzmann's constant, $T$ is temperature, and $v_{0}$ is assumed to be of the order of $10^{13}$. Although $v_{0}$ can be determined more exactly if required, ${ }^{27}$ it is common practice to use a fixed preexponential factor of the order of $10^{13} .^{26,28}$ This value agrees with calculations done for single atom hops of $\mathrm{Ag}$ on a $\mathrm{ZnO}$ substrate showing that the pre-factors are typically between $0.3 \times 10^{13}$ and $14 \times 10^{13}$. The reasoning for using a fixed value is that a more exact value can be very computational expensive to determine and the frequency of events from the Arrhenius equation depends more strongly on the value of $E_{\mathrm{B}}$ than $v_{0}$. Typically 200-1000 saddle point searches were conducted per defect volume to find a set of transitions.

\section{B. Lat-AKMC}

A lattice-based adaptive KMC method (Lat-AKMC) was also developed to access long time scales on larger systems. The method makes the approximation that all atoms are positioned on a lattice. This approach can speed up simulations by over two orders of magnitude compared to the off-lattice method. Lat-AKMC is currently limited to running efficiently on a single processor but can conduct 100,000's of KMC steps within a fewdays on modern machines.

The general lattice based AKMC algorithm for a hexagonal lattice, such as the $\mathrm{ZnO}(000 \overline{1})$ and $\mathrm{Ag}(111)$ surfaces, is largely similar to the off-lattice method apart from the method of conducting the transitions searches. Searches are run on each under-coordinated atom in the system as follows:

(i) Step 1: Find potential transition sites. For a given under co-ordinated atom, move said atom in six directions on the current layer, six directions on the layer above and six directions on the layer below to gain a list of potential transition sites.

(ii) Step 2: Remove known bad transition sites. Determine if each transition site is an accepted location and disregard non accepted transition sites such as intersecting atoms or other known highly unstable sites which may be system dependent.

(iii) Step 3: Create graph keys. Calculate the graph keys (using NAUTY) corresponding to the local environment (all atoms within $\approx 5 \mathrm{NN}$ distance from the under co-ordinated atom) of the initial state and of each final transition site.

(iv) Step 4: Reuse transitions. Check if transitions have been found before with the same initial and final graph keys. If so, use the energy barrier associated with the transition and add the final state and energy barrier to the $\mathrm{KMC}$ event list.

(v) Step 5: Minimise states. For any remaining transitions, minimise the initial state and each remaining final state using the conjugate gradient method.

(vi) Step 6: Check atoms are in lattice sites. Check that all atoms within the minimised states have not significantly moved from their corresponding lattice sites. If atoms have moved away from lattice sites, the method may not be appropriate for the current system and the off-lattice method may have to be used to further the simulation.

(vii) Step 7: Calculate barriers. Find the transition energy barrier between the minimised initial and final transition states using the NEB method and add to the KMC event list.

(viii) Step 8: Store transitions. Store the initial and final state graph keys along with the energy barriers of any new transitions found for reuse.

For the $\mathrm{Ag} / \mathrm{ZnO}$ system, possible lattice sites, labeled $\mathbf{A}, \mathbf{B}$, and $\mathbf{C}$, are shown in Fig. 1. The first layer $\mathrm{Ag}$ adatoms can move in three different directions in surface plane-from $\mathbf{A}$ to $\mathbf{C}$ sites or vice versa. The other three directions are disallowed as $\mathbf{B}$ sites above surface $\mathrm{O}$ 


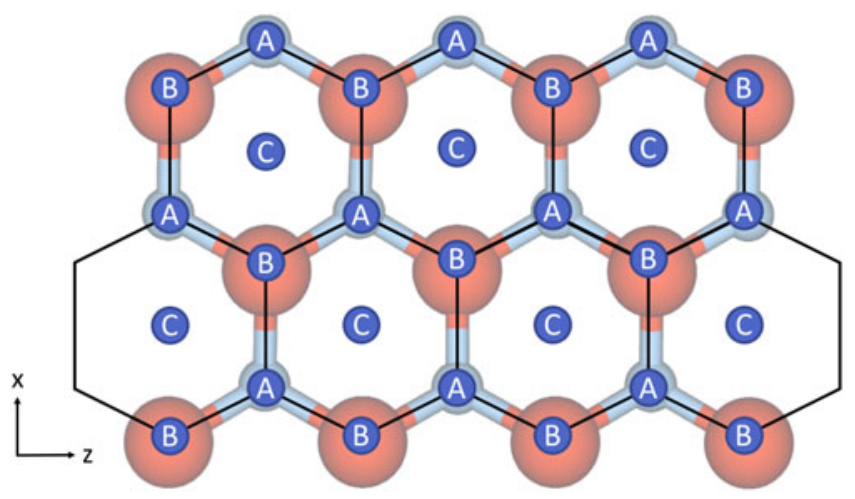

FIG. 1. Schematic of the hexagonal lattice associated with the polar $\mathrm{ZnO}$ surface and possible sites for atoms to reside in. Sites labeled with $\mathbf{A}$ correspond to the position in the lattice occupied by $\mathrm{Zn}$ atoms, sites labeled with $\mathbf{B}$ refer to $\mathrm{O}$ positions and sites labeled with $\mathbf{C}$ refer to hollow sites where first layer $\mathrm{Ag}$ ad-atoms prefer to be situated.

atoms are unstable. Adatoms can also jump up to the second Ag layer. A second layer adatom can move in three directions on the Ag plane to stable lattice sites, typically from A to $\mathbf{B}$ sites or vice versa but can vary depending on the first layer Ag stacking. Diffusion can also be extended to further layers. There is also the possibility to jump down or up a layer if appropriately sited.

With this method it was possible to simulate growth of $\mathrm{Ag}$ on $\mathrm{ZnO}$ surfaces containing 432 atoms for times up to $0.08 \mathrm{~s}$. In Lat-AKMC the MD part of the simulation is replaced by randomly placing an $\mathrm{Ag}$ atom on a stable lattice site. This is a good approximation as single point deposition simulations ${ }^{9}$ showed that no $\mathrm{Ag}$ atoms penetrate or damage the $\mathrm{ZnO}$ surface at low deposition energies and come to rest in stable lattice sites. Single atoms can diffuse around the surface, but concerted motion of clusters was not implemented, although some cluster motion was observed as a series of single atom moves. This is a good approximation for this system since the off-lattice AKMC results showed few concerted motions of clusters.

Although only single atom moves are allowed in the simulation, the energy barriers for diffusion of the atoms were determined adaptively by the nudged elastic band method $^{25}$ on a large local environment rather than from a pre-defined event list ${ }^{29}$ or by counting bonds within a small region. ${ }^{30}$ The local environment (defect volume) size is chosen such that any atoms that move outside of the region have a negligible effect $(<0.01 \mathrm{eV})$ on barrier heights of the atom considered for the move. For the $\mathrm{Ag} / \mathrm{ZnO}$ system, this region (defect volume) is chosen to be a radius of over $5 \mathrm{NN}$ (5.9 $\AA$ ) surrounding an under co-ordinated atom. In Lat-AKMC, nearby defect volumes are not combined as only single atom hops are considered. Transitions are stored so that when a local configuration/defect volume is one that has already occurred in the simulation, the transitions are not recalculated but reused - as with the off-lattice method. However with the Lat-AKMC method, only the graph keys of the initial and final states and corresponding transition energy barriers are stored, not all atom positions within the defect volume. A transformation matrix is not used for the on-lattice based transition re-use algorithm. Instead, if the initial state's defect volume and transitions have previously been stored, all possible transitions are still considered and final state graph keys are calculated. If the final graph keys exists in the stored transitions list, the transition energy barrier is used and the NEB method is not used to find the transition pathway.

Finally a superbasin method is used when transition barriers are small. The superbasin basin treats states connected by low energy barriers as a single objecta superbasin. The rate to leave a superbasin is then calculated from the total average occupancy time in each state within the superbasin by using the mean rate method. ${ }^{15}$ Although there are other methods for calculating the rate in which to leave the superbasin, such as using absorbing Markov chains, ${ }^{31}$ the mean rate method combined with the way the superbasins are constructed within Lat-AKMC allows a system to escape a superbasin before all states within that superbasin have been explored. Each superbasin is built on-the-fly as each low energy barrier transition is found. During the construction of superbasins, the final state of a potential event is not in the built superbasin yet, thus the rates for these transitions are calculated the same way as the escaping transitions and included in the KMC event list. This implies it is possible at each step to escape the superbasin before all the states connected by low-energy barriers are explored, which may improve computational efficiency when particularly large superbasins exist. The need for a superbasin method to accelerate a simulation arises when small transition energy barriers dominate and no net diffusion or deposition events are likely to occur. For our system, small energy barriers dominate mainly when $\mathrm{Ag}$ atoms deposit on top of existing $\mathrm{Ag}$ atoms. The superbasin method was relatively simple to implement on the lattice but was not used in the off-lattice AKMC simulations for this system as small differences of atom positions can alter the canonical label (graph key) given to the local environment despite the state being essentially identical. However, the superbasin method has been used for other systems using the off-lattice AKMC method. ${ }^{17}$

\section{RESULTS}

\section{A. Single point depositions}

As a method of testing the model, single point deposition simulations were conducted. Both the simplified pair potential model and the many-body ReaxFF model 
were used and compared. Via $\mathrm{MD}$, single $\mathrm{Ag}$ atoms and $\mathrm{Ag}$ dimers were deposited normal to a perfect, O-terminated, $\mathrm{ZnO}(000 \overline{1})$ surface at a range of energies and the final resting positions of deposited atoms were analyzed (Table I). A period of 10 ps was given for deposited atoms to hit the surface and excess energy to dissipate from the target region.

The simplified model suggests that $40 \%$ of deposited $\mathrm{Ag}$ atoms penetrate the $\mathrm{ZnO}$ surface when deposited at $3 \mathrm{eV}$. In contrast, the ReaxFF model predicts no $\mathrm{Ag}$ atoms would penetrate the surface at these energies. ${ }^{9}$ In fact, the deposition energy of $\mathrm{Ag}$ atoms has been as high as $10 \mathrm{eV}$ before any ad-atoms penetrate the surface. The marked difference between the single point deposition results is due to the simplified model over estimating the stability of interstitial $\mathrm{Ag}$ atoms in the $\mathrm{ZnO}$ substrate.

Single point deposition simulations can provide an insight into ideal deposition energies and preferred adsorption sites. The ReaxFF model results suggest that $\mathrm{Ag}$ ad-atom prefer to adsorb to sites on the surface directly above a hollow or a $\mathrm{Zn}$ atom on the O-terminated surface. These preferred adsorption sites are also shown to be key in growth mechanisms during the AKMC growth simulations and are exploited for faster Lattice AKMC simulations.

Due to the conflict between the results obtained using the simplified model and the ab initio data and large differences observed in deposition simulations, the more comprehensive ReaxFF potential was chosen for further investigations into thin film growth despite being more computationally expensive.

\section{B. Transition barriers}

Before carrying out the KMC simulations, the diffusion involving clusters of up to three atoms was considered to determine approximate transition times. A single $\mathrm{Ag}$ atom has two potentially stable sites on the $\mathrm{ZnO}$ surface, which lie either directly above a $\mathrm{Zn}$ atom or above a vacant site in the next layer. These are shown in Fig. 2(a) together with the associated transition barriers between the two states and their related hop times at $300 \mathrm{~K}$. In Fig. 2(b), the barriers for an Ag dimer to form and separate are given. It can be seen that $\mathrm{Ag}$ does not prefer to become a dimer as the energy barrier to form is larger than the barriers to split and move away. This is

TABLE I. The outcomes of single point depositions of single Ag atoms and $\mathrm{Ag}_{2}$ dimers on $\mathrm{ZnO}$ at $3 \mathrm{eV}$. A marked difference between the two models is shown. The ReaxFF deposition results are from Ref. 9.

\begin{tabular}{lccrrr}
\hline \hline & \multicolumn{2}{c}{ Simplified model } & & \multicolumn{2}{c}{ ReaxFF } \\
\cline { 2 - 3 } \cline { 5 - 6 } & $\mathrm{Ag}$ & $\mathrm{Ag}_{2}$ & & $\mathrm{Ag}$ & $\mathrm{Ag}_{2}$ \\
\hline Penetrate (\%) & 39.00 & 42.50 & & 0.00 & 0.00 \\
Adsorb (\%) & 61.00 & 57.50 & & 100.00 & 100.00 \\
\hline \hline
\end{tabular}

also true for other metastable configurations of $\mathrm{Ag}$ dimers on the $\mathrm{ZnO}$ surface. However Fig. 2(c) shows that if an $\mathrm{Ag}$ trimer forms on the surface this is a very stable structure. Further calculations showed that the barriers for a single adatom to join an existing trimer were also higher than the barriers to move further away. In general, for small clusters of $\mathrm{Ag}$ atoms the energy barriers for additional $\mathrm{Ag}$ adatoms to move further away from existing clusters are lower than for them to join the cluster.

Thus the Ag atoms do not initially want to cluster on the surface. This was borne out by initial growth simulations which showed that atoms deposited onto a perfect surface would spend the first few microseconds avoiding each other until sufficient silver covered the surface so they could no longer avoid it.

Adatom diffusion barriers were also calculated when one or two layers of $\mathrm{Ag}$ were already present on the $\mathrm{ZnO}$ surface. In this case, the possible absorption sites are above a $\mathrm{Zn}$ atom in the layer $\mathrm{ZnO}$ surface or above an $\mathrm{O}$ atom for the first Ag layer. The relative diffusion barriers and Ehrlich-Schwoebel barriers to drop down to the layers below are shown in Table II. (These are actual barriers rather than the differences in binding energy.) It can be seen that the barriers to diffuse around the first layer are smaller than the barriers to escape to the layer below. This is even more pronounced for the second silver layer. Previous simulations of $\mathrm{Ag}$ growth on $\mathrm{Ag}$ have also shown that the diffusion barriers in the layers are smaller than the barriers to drop down although with a smaller relative difference than calculated here. ${ }^{12}$

\section{AKMC simulations on a perfect $\mathrm{ZnO}(000 \overline{1})$ surface}

Because the initial simulations showed that the $\mathrm{Ag}$ atoms prefer to be separated, to enhance the speed of the simulations, $16 \mathrm{Ag}$ atoms were placed randomly separated on $\mathrm{ZnO}$ before the growth simulations were initiated. With the high concentration of $\mathrm{Ag}$ atoms on the surface, clusters are forced to form and the simulation runs more quickly. The simulation starts with wellseparated adatoms that then cluster together, followed by additional Ag atoms being deposited. Typically, the newly deposited atoms diffuse around the surface until they attach onto an existing cluster. This is then followed by another deposition event. Figure 3(b) shows the state of the development of the growth after $122 \mathrm{~ms}$ with a deposition rate of $12 \mathrm{~mL} / \mathrm{s}$ at $300 \mathrm{~K}$. It is possible to see that the second Ag layer has formed before the first layer completely covers the underlying surface.

Our previous work has indicated that AKMC simulations are necessary to model growth because of a large number of unexpected concerted motions that can occur during the growth process. In the case of $\mathrm{Ag}$ growth on $\mathrm{Ag}$, it was these concerted motions that could explain the 
(a)

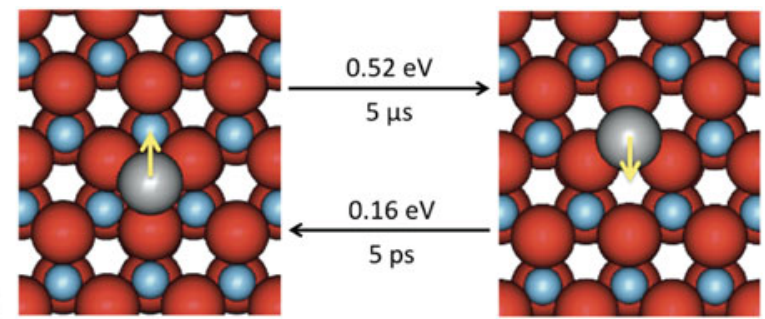

(b)

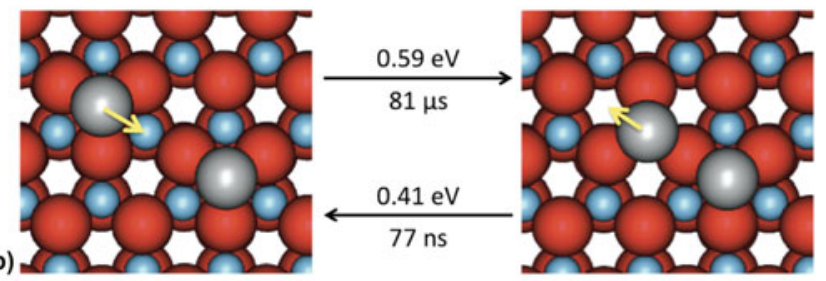

(c)

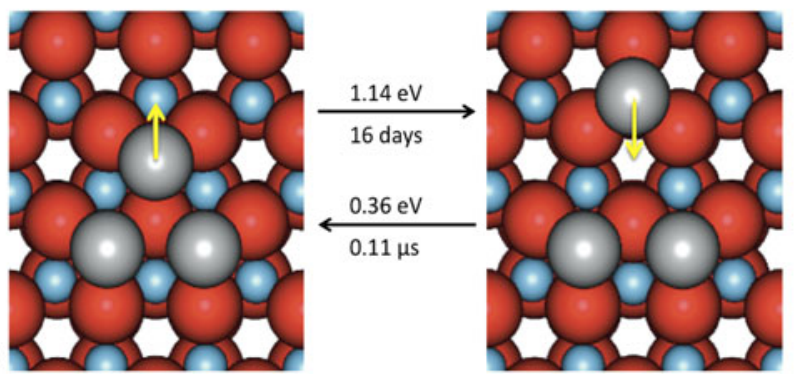

FIG. 2. (a) Single Ag adatom transitions on a perfect $\mathrm{ZnO}(000 \overline{1})$ surface. Hop times are calculated at $300 \mathrm{~K}$. (b) Ag dimer forming and breaking transitions. (c) Ag trimer breaking transitions. Black arrows show transitions from the state on the left (right) to the state on the right (left) with corresponding energy barriers. Yellow arrows indicate the direction of the transition. Oxygen atoms are colored in red, zinc atoms in blue and silver atoms in silver. All images are shown as if one is looking directly down at the surface. (For interpretation of the reference to color in this figure legend, the reader is referred to the web version of this article.)

formation of twin boundaries ${ }^{12}$ and in the case of $\mathrm{ZnO}$ growth how chains of atoms on the surface could move concertedly to form into hexagons. ${ }^{13}$ It was therefore surprising to discover that as the silver deposits, there were only a few examples of concerted motion. One such

TABLE II. The energy barriers for an adatom to diffuse over the surface compared to the barriers to drop down to the layer below. ${ }^{32}$ The stacking sequence definition is A: $\mathrm{Zn}$ layer, $\mathrm{B}: \mathrm{O}$ layer, c:Ag first layer (with the $\mathrm{Ag}$ arranged as on the left hand images of Fig. 2) and b:Ag second layer above the $\mathrm{O}$ atoms.

\begin{tabular}{|c|c|c|c|c|c|}
\hline \multicolumn{3}{|c|}{ First Ag layer-ABc stacking } & \multicolumn{3}{|c|}{ Second Ag layer-ABcb stacking } \\
\hline Initial & Final & $E_{\mathrm{B}}(\mathrm{eV})$ & Initial & Final & $E_{\mathrm{B}}(\mathrm{eV})$ \\
\hline Above $\mathrm{Zn}$ & Above $\mathrm{O}$ & 0.17 & Above Ag & Above $\mathrm{Zn}$ & 0.01 \\
\hline Above $\mathrm{O}$ & Above $\mathrm{Zn}$ & 0.30 & Above $\mathrm{Zn}$ & Above Ag & 0.06 \\
\hline Above $\mathrm{Zn}$ & Drop down & 0.35 & Above Ag & Drop down & 0.75 \\
\hline Above $\mathrm{O}$ & Drop down & 0.72 & Above $\mathrm{Zn}$ & Drop down & 0.89 \\
\hline
\end{tabular}

example is shown in Fig. 3(a). As can be seen the energy barrier for the two atoms to move together is very similar to that for the single atom move. In both cases, the corresponding reverse barriers are $>1 \mathrm{eV}$ and are unlikely to occur in AKMC timescales. Isolated $\mathrm{Ag}$ dimers tend to split and the trimers having formed as equilateral triangles are effectively pinned and do not move. Since concerted motions do not appear to be important mechanisms in the growth of the first Ag layer, this observation also motivated us to develop the Lat-AKMC code based on single atom moves to access larger systems.

\section{AKMC simulations on a $\mathrm{ZnO}(000 \overline{1})$ surface with oxygen vacancies and step edges}

In our previous work modeling the growth of $\mathrm{ZnO}$ in reactive magnetron sputtering, it was shown that parts of the surface could be oxygen deficient. ${ }^{13}$ In addition, growth does not always occur uniformly and step edges can be present on any surface. It was found that it was $0.87 \mathrm{eV}$ more favourable for an $\mathrm{Ag}$ atom to sit on the surface away from a single vacancy rather that bind into the vacant $\mathrm{O}$ atom position. During the growth process, silver atoms initially avoid the region near to the vacancy, but eventually a layer of silver forms over the surface as shown in Fig. 4. Also shown in the figure is the case where multiple $O$ vacancies are placed together and the surface reconstructs to a relaxed triangular region. This type of structure was found in our previous growth simulations of $\mathrm{ZnO}^{13} \mathrm{Ag}$ atoms avoid the triangular structure and form clusters around it. The occasional $\mathrm{Ag}$ atom diffuses into this region but rapidly leaves and joins the defect free part of the surface.

In contrast to $\mathrm{O}$ vacancies, $\mathrm{Ag}$ atoms prefer to bind to step edges. Such positions are around $0.2 \mathrm{eV}$ more favourable than if the $\mathrm{Ag}$ atom was located in isolation on a perfect surface. At an edge, an atom can climb up a step edge onto the upper surface with a high transition energy of $1.01 \mathrm{eV}$, the reverse barrier being 0.87 $\mathrm{eV}$. Such events are unlikely to occur at room temperature.

\section{E. Lat-AKMC simulations on a perfect $\mathrm{ZnO}(000 \overline{1})$ surface}

The Lat-AKMC model reproduces the growth mechanisms observed in the off-lattice approach. Initially, a substrate of the same dimensions in the $x$ and $z$ directions $(2.28 \times 2.63 \mathrm{~nm})$ of that used in the offlattice model was considered. Initial growth mechanisms show that single adatoms diffuse readily across the surface and that Ag dimers can form and split at similar rates (with transition barriers typically between 0.4 and $0.6 \mathrm{eV})$. As with the off-lattice method, once clusters of three or more $\mathrm{Ag}$ adatoms start to form, the energy barriers to escape the cluster become larger and so 3 atom 
clusters act as nucleation sites on the surface. Small clusters form initially before joining together via single atoms strings. Once atoms begin to deposit on existing $\mathrm{Ag}$ clusters, the $\mathrm{Ag}$ atoms are very mobile but when second layer clusters have formed they can diffuse as a series of single atom moves. An example is shown in Fig. 5 where $6 \mathrm{Ag}$ atoms change stacking.

The lowest energy barriers for diffusion on the first silver layer are 0.17 and $0.3 \mathrm{eV}$. These are lower than the relative barriers to drop down to the first layer-see Table II or the equivalent barrier for deposition $(0.59 \mathrm{eV}$ on a 100 atom surface), so these are handled using a superbasin method ${ }^{15}$ which considerably speeds up the simulation.
Using the lattice based method it was possible to simulate the deposition of over $400 \mathrm{Ag}$ atoms in the larger system on a perfect $\mathrm{ZnO}(000 \overline{1})$ substrate as seen in Fig. 6. As the superbasin method is used within the method, the lattice-based method runs a lot quicker and can push through dominating small energy barrier transitions. Initially, only $1 \mathrm{Ag}$ ad-atom is placed on the surface in contrast to the saturated surface required for the off-lattice method to see small clusters form. Subsequently, the on-lattice method allows further $\mathrm{Ag}$ atoms deposit and clusters form with a simulated deposition rate of $12 \mathrm{~mL} / \mathrm{s}$ at $300 \mathrm{~K}$. The second layer of $\mathrm{Ag}$ atoms begins to form before the first layer is completely covered. Parts of the original $\mathrm{ZnO}$ surface are visible as sets of linked
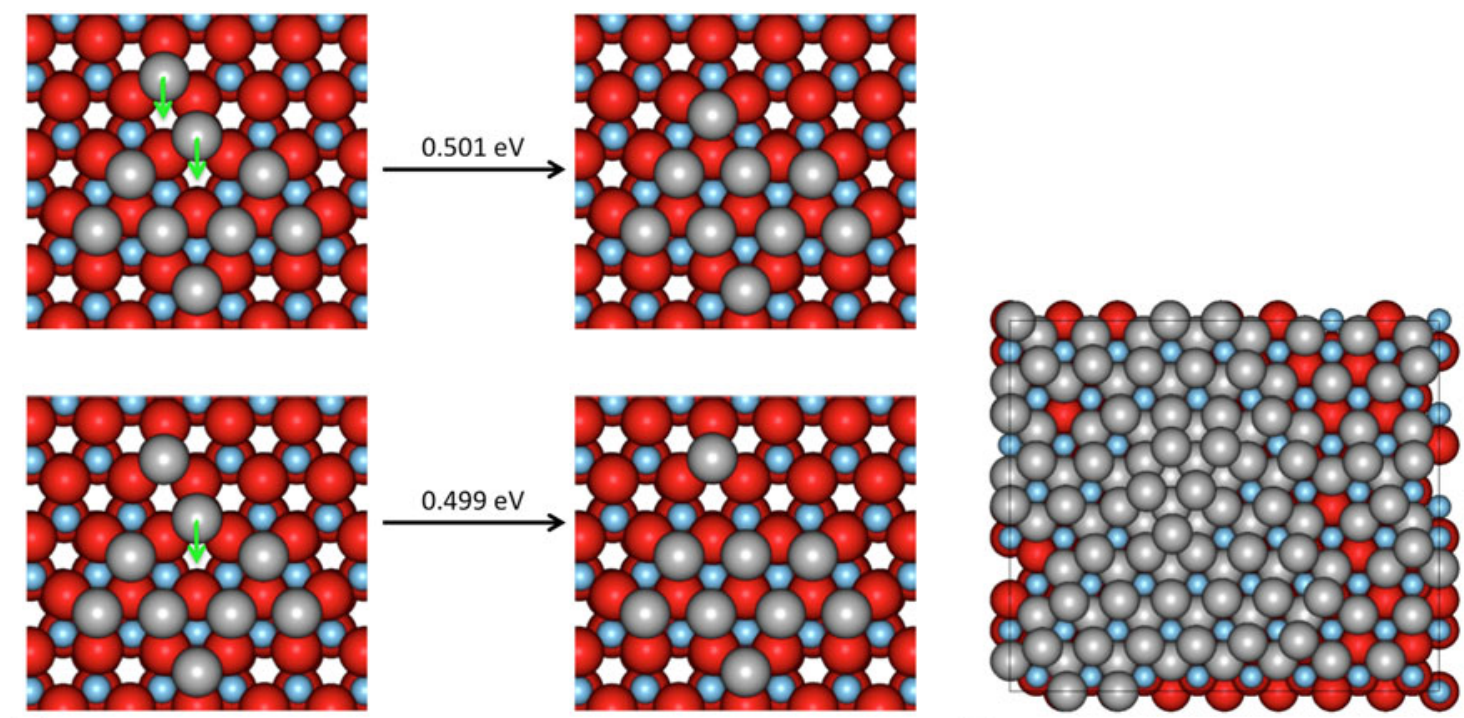

(a)

(b)

FIG. 3. (a) An example of concerted motion where the two-atom move has effectively the same barrier as the single atom move. (b) The growth after $122 \mathrm{~ms}$ showing the formation of the second layer of $\mathrm{Ag}$ atoms before the $\mathrm{ZnO}$ surface has been completely covered.
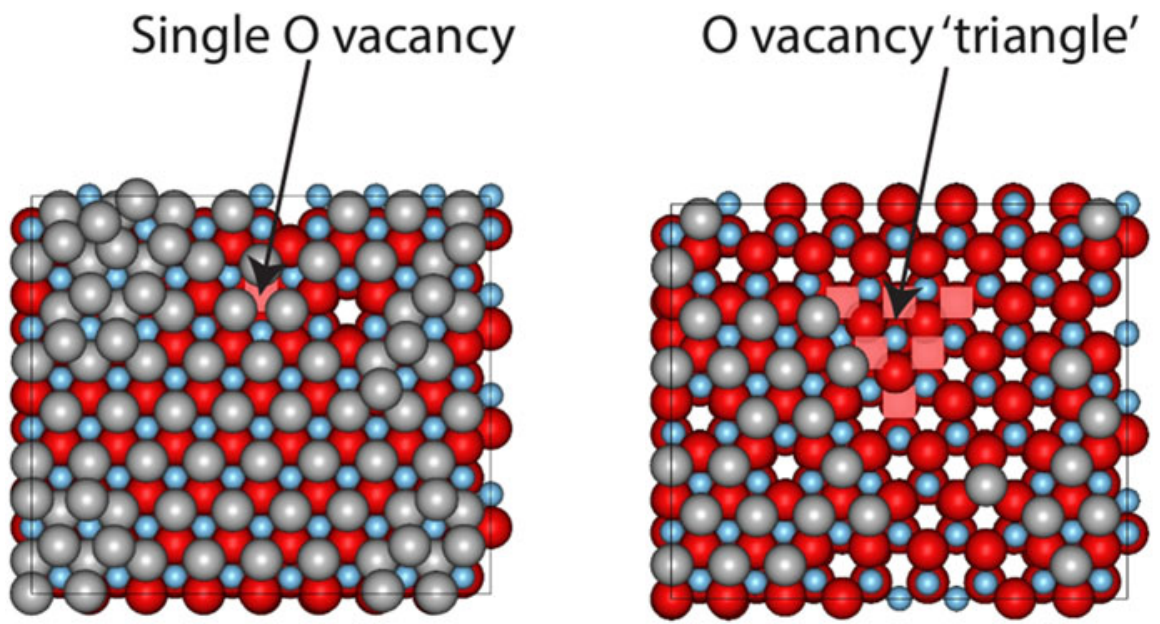

FIG. 4. Ag growth when oxygen vacancies occur in the $\mathrm{ZnO}$ surface. The left hand image is after $0.11 \mathrm{~s}$ of growth time and the right hand image after $0.022 \mathrm{~s}$. The deposition rate was $12 \mathrm{~mL} / \mathrm{s}$ oxygen vacancies are indicated by pink squares. 
chains. As can be seen from Table II the barriers for the second layer Ag atom to drop down to the first Ag layer is much larger than the barrier from the first layer to the $\mathrm{ZnO}$ surface and so second layer clusters form.

At this stage of growth, it is clear that it is difficult to obtain a complete first layer of $\mathrm{Ag}$ growth with large sections of the original surface left uncovered. On top of this, growth simulations imply that growth of the second Ag layer is even less likely to produce a complete and connected surface. In the example shown in Fig. 6, 1 monolayers worth of deposited $\mathrm{Ag}$ only covers $78 \%$ of the original $\mathrm{ZnO}$ surface. Results, therefore, suggest that $\mathrm{Ag}$ growth on $\mathrm{ZnO}$ will produce islands rather than smooth layer by layer growth.

In experiments, island growth of $\mathrm{Ag}$ on $\mathrm{ZnO}$ has been observed $^{4}$ when $\mathrm{Ag}$ has been deposited at low energies via evaporation and once a few nanometres have grown. However, little is known about the formation of islands and growth mechanisms experimentally for the initial one or two layers of growth. Our simulations show Ag islands forming leaving chains of the original $\mathrm{ZnO}$ surface exposed. Currently to extend the calculations to investigate how the islands further develop requires an excessive amount of computational time. But it might be expected that $\mathrm{Ag}$ islands would continue to form as on the pure $\mathrm{Ag}$ surface.

\section{F. Island growth on a perfect $\mathrm{ZnO}(000 \overline{1})$ surface}

By combining the rate at which particles arrive at the surface with transition rates of atoms dropping down from an existing island it is possible to predict the critical island size assuming all first layer islands are centrosymmetric (hexagonal). The definition of a critical island size in this sense is how large the island is likely to grow before the islands start to form on the layer above it. This method treats all possible adsorption sites for a lone $\mathrm{Ag}$ adatom on an existing $\mathrm{Ag}$ island as a single superbasin and transitions for the $\mathrm{Ag}$ adatom to drop down from on top of the island and join the cluster as the escaping mechanisms as reported in Ref. 32. The island sizes are extremely sensitive to temperature and the size of the barriers. For example, for a deposition rate of $12 \mathrm{~mL} / \mathrm{s}$, this is less than 350 atoms at room temperature whereas for higher temperatures, critical island sizes can be in excess of 500 atoms if $\mathrm{Ag}$ grows in uniform hexagonal structures.

Again if we treat the adsorption sites on an island as a single superbasin, the mechanisms of island growth can be explored further. The probability of an adatom being located at any single site in the superbasin before escaping and joining the layer below, i.e., growing in the surface plane direction, is calculated by normalising the mean residence time in each site with respect to the total residence time within the basin. When considering symmetric (hexagonal) islands, results suggest that for more than $98 \%$ of the time in the island superbasin, the

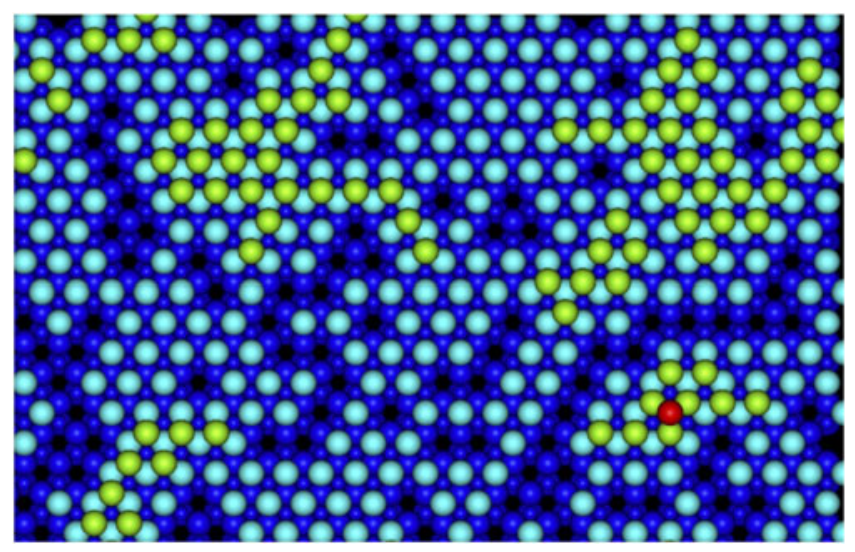

FIG. 6. An example growth simulation after $77 \mathrm{~ms}$ of simulation time with $428 \mathrm{Ag}$ atoms deposited (equivalent to 1 monolayers worth); 87 atoms are in the second layer of $\mathrm{Ag}$ with a single $\mathrm{Ag}$ atom in the third layer. Here dark blue spheres depict the $\mathrm{ZnO}$ substrate (small spheres are $\mathrm{Zn}$ and large spheres are $\mathrm{O}$ ); light blue spheres are the first layer Ag atoms, green spheres second layer $\mathrm{Ag}$ atoms and the red sphere is a third layer Ag atom.
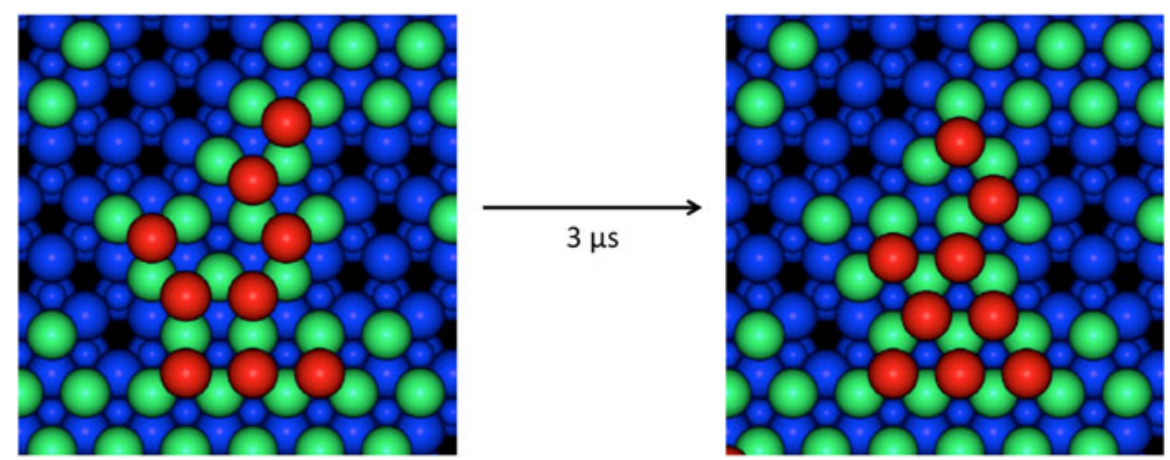

FIG. 5. Example of $6 \mathrm{~s}$ layer Ag atoms shifting from ABca to ABcb stacking sites (see the caption to Table I for a definition) during a Lat-AKMC simulation. The total process takes around $3 \mu$ s to complete which is equivalent to a single transition barrier of $0.45 \mathrm{eV}$. In this figure, atoms are colored by height. The underlying $\mathrm{ZnO}$ lattice is shown in blue with the $\mathrm{Zn}$ atoms as the small circles. The first layer $\mathrm{Ag}$ atoms are green and the second layer red. 
$\mathrm{Ag}$ is situated directly above an $\mathrm{O}$ atom. Preferable sites are also located toward the edges of the island with the most favourable sites located at the corner and above $\mathrm{O}$ atoms (see Fig. 7).

The probabilities for atoms to drop off the island in certain directions are considered. The rates for Ag atoms to escape the island superbasin when situated above $\mathrm{Zn}$ and $\mathrm{O}$ edges sites are $1.32 \times 10^{7}$ and 8.02 times per second respectively. By combining these rates with the residence probabilities shown in Fig. 7, it suggests that $\mathrm{Ag}$ atoms are $\approx 10^{4}$ times more likely to escape the island superbasin in directions that have adsorption sites directly above $\mathrm{Zn}$ surface atoms at the edges. This implies that $\mathrm{Ag}$ islands would not grow in regular hexagonal or circular shapes but rather in long chains and agrees with LatAKMC growth simulation results (Fig. 6).

\section{DISCUSSION AND CONCLUSIONS}

Using a combination of multi-time scale methods we have identified the main mechanisms that are responsible for $\mathrm{Ag}$ growth on the $\mathrm{ZnO}(000 \overline{1})$ surface. Unlike previous investigations using these techniques there is little evidence of concerted motions in the growth of the first $\mathrm{Ag}$ layer. If there are step edges on the $\mathrm{ZnO}$ surface $\mathrm{Ag}$ prefers to attach there but the barriers to move up or drop down are large. Ag also prefers to bond away from $\mathrm{O}$ vacancies in the surface but eventually does cover such areas as the surface coverage increases. The calculated Ehrlich-Schwoebel (ES) barriers are generally higher than those previously calculated for $\mathrm{Ag}$ growth on $\mathrm{Ag}(0.42 \mathrm{eV})^{12}$ varying between 0.35 and $0.89 \mathrm{eV}$. These values are much higher than the corresponding values for diffusion over the surface indicating that island growth is more likely than layer by layer growth and this is borne out by the Lat-AKMC simulations.

The lattice approximation in the Lat-AKMC method manages to drive growth simulations past those achieved using the off-lattice approach. By considering larger systems, Lat-AKMC simulations produced a better understanding of how first layer Ag islands interact with each other and how the islands initially grow.

A mean rate method was applied to $\mathrm{Ag}$ diffusion on a first layer island within the on-lattice system and indicates that $\mathrm{Ag}$ is likely to drop of any existing islands at corners. This agrees with Lat-AKMC growth simulations and implies that islands will not grow in uniform circles but rather in long chains.

Previous work ${ }^{9}$ has shown that the $\mathrm{Ag}$ deposition energy can be increased to around $10 \mathrm{eV}$ before the $\mathrm{Ag}$ atoms penetrate below the surface and mixing occurs at the interface. Thus if it is required to have as sharp an interface as possible then the deposition energy should be kept below this value. On the other hand, simulations of

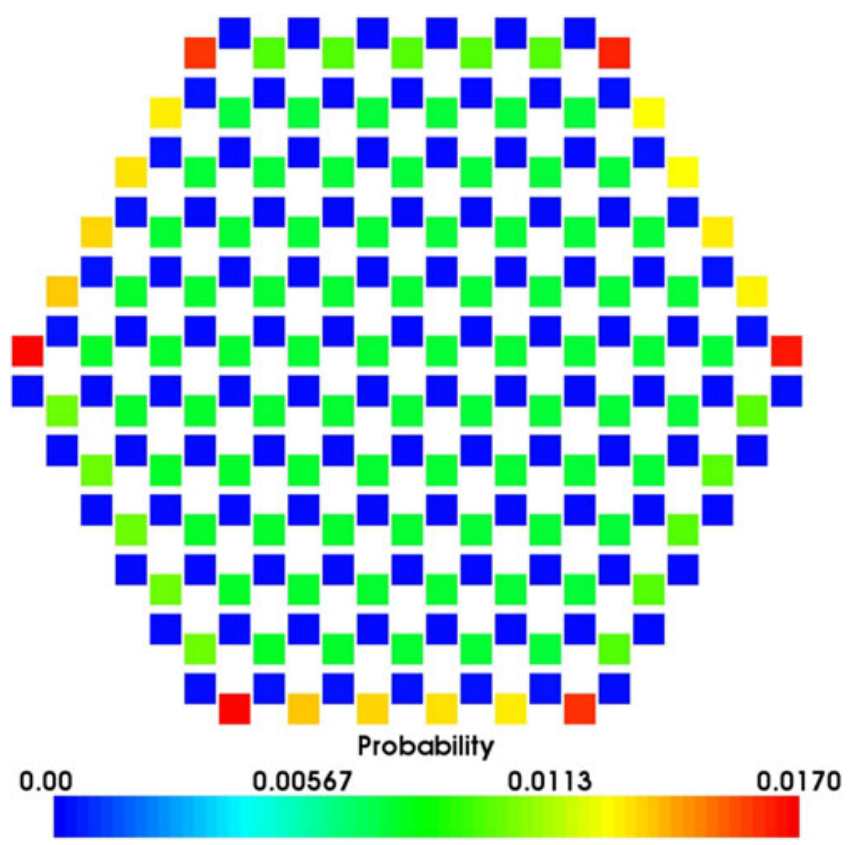

FIG. 7. Probability heat map of a single Ag adatom being in each site above a hexagonal island before dropping off and joining the layer below. The island consists of $127 \mathrm{Ag}$ atoms on a perfect $\mathrm{ZnO}(000 \overline{1})$ surface in a favourable $\mathrm{ABc}$ stacked configuration. Each square denotes a lattice site considered within the island superbasin. Blue sites correspond to adsorption sites directly above surface $\mathrm{Zn}$ atoms whilst the favourable green, yellow and red sites are directly above surface $\mathrm{O}$ atoms.

the growth of $\mathrm{Ag}$ on $\mathrm{Ag}$ have shown that smoother films with fewer defects can be produced by increasing the deposition energy to between 30 and $40 \mathrm{eV}$ but this causes considerable mixing at the interface between the substrate and the added atoms. One strategy for minimising island growth, while maintaining a sharp interface, might be to increase gradually the deposition energy during growth.

The development of the underlying modeling techniques used here is continuing so that the AKMC technique will be able to handle larger and more complex systems for longer experimental times. Further advances within the on lattice AKMC methodology could widely extend the range of its applications to include modeling more complicated growth mechanisms and allowing higher energy deposition events that may damage the substrate. This could be done by implementing concerted motion transitions on clusters and running MD for deposition events/collision cascades followed by repositioning atoms on lattice sites. Nonetheless the current state of these long time scale methods is such that insight can be provided into many important growth processes that are inaccessible to experiment. This helps understand the underlying scientific concepts as well as providing guidelines as to the best conditions required to optimise film performance. 


\section{ACKNOWLEDGMENTS}

We acknowledge EPSRC (Grant numbers EP/ K000055/1 and EP/M018210/1) and AGC Glass Europe for partial funding. The computational work was also supported by HPC Midlands. We also acknowledge Ying Zhou from Loughborough University and David Cornil, David Beljonne, and Jérôme Cornil from the University of Mons for useful discussions. The underlying research materials are available on request.

\section{REFERENCES}

1. R.E. Hummel and K.H. Guenther: Handbook of Optical Properties: Thin Films for Optical Coatings, Vol. 1 (CRC Press, Boca Raton, Florida, 1995).

2. H.J. Glaser: History of the development and industrial production of low thermal emissivity coatings for high heat insulating glass units. Appl. Opt. 47, C193 (2008).

3. D. Cornil, H. Wiame, B. Lecomte, J. Cornil, and D. Beljonne: Which oxide for low-emissivity glasses? First-principles modeling of silver adhesion. ACS Appl. Mater. Interfaces 9, 18346 (2017).

4. E. Duriau, S. Agouram, C. Morhain, T. Seldrum, R. Sporken, and

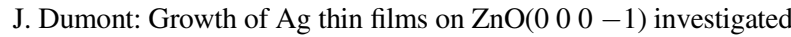
by AES and STM. Appl. Surf. Sci. 253, 549 (2006).

5. Z. Lin and P.D. Bristowe: Microscopic characteristics of the $\mathrm{Ag}(111) / \mathrm{ZnO}(0001)$ interface present in optical coatings. Phys. Rev. B 75, 205423 (2007).

6. I. Gheewala, S.D. Kenny, and R. Smith: Atomistic-scale modelling of nanoindentation into optical coatings. Philos. Mag. 89, 3499 (2009).

7. K. Choudhary, T. Liang, A. Chernatynskiy, S.R. Phillpot, and S.B. Sinnott: Charge optimized many-body (COMB) potential for $\mathrm{Al}_{2} \mathrm{O}_{3}$ materials, interfaces, and nanostructures. J. Phys.: Condens. Matter 27, 305004 (2015).

8. A.C.T. van Duin, S. Dasgupta, F. Lorant, and W.A. Goddard: Reaxff: A reactive force field for hydrocarbons. J. Phys. Chem. A 105, 9396 (2001).

9. A. Lloyd, D. Cornil, A.C.T. van Duin, D. van Duin, R. Smith, S.D. Kenny, J. Cornil, and D. Beljonne: Development of a ReaxFF potential for $\mathrm{Ag} / \mathrm{Zn} / \mathrm{O}$ and application to $\mathrm{Ag}$ deposition on $\mathrm{ZnO}$. Surf. Sci. 645, 67 (2016).

10. G. Henkelman and H. Jónsson: Long time scale kinetic Monte Carlo simulations without lattice approximation and predefined event table. J. Chem. Phys. 115, 9657 (2001).

11. C. Scott, S. Blackwell, L. Vernon, S. Kenny, M. Walls, and R. Smith: Atomistic surface erosion and thin film growth modelled over realistic time scales. J. Chem. Phys. 135, 174706 (2011).

12. S. Blackwell, R. Smith, S.D. Kenny, and J.M. Walls: Modeling evaporation, ion-beam assist, and magnetron sputtering of thin metal films over realistic time scales. Phys. Rev. B 86, 035416 (2012).

13. S. Blackwell, R. Smith, S.D. Kenny, J.M. Walls, and C.F. SanzNavarro: Modelling the growth of $\mathrm{ZnO}$ thin films by PVD methods and the effects of post-annealing. J. Phys.: Condens. Matter 25, 135002 (2013).

14. K.A. Fichthorn and Y. Lin: A local superbasin kinetic Monte Carlo method. J. Chem. Phys. 138, 164104 (2013).

15. B. Puchala, M.L. Falk, and K. Garikipati: An energy basin finding algorithm for kinetic Monte Carlo acceleration. J. Chem. Phys. 132, 134104 (2010).

16. L.K. Béland, P. Brommer, F. El-Mellouhi, J-F. Joly, and N. Mousseau: Kinetic activation-relaxation technique. Phys. Rev. E 84, 046704 (2011).

17. A.L. Lloyd, Y. Zhou, M. Yu, C. Scott, R. Smith, and S.D. Kenny: Reaction pathways in atomistic models of thin film growth. J. Chem. Phys. 147, 152719 (2017).

18. H.J.C. Berendsen, J.P.M. van Postma, W.F. van Gunsteren, A. DiNola, and J.R. Haak: Molecular dynamics with coupling to an external bath. J. Chem. Phys. 81, 3684 (1984).

19. N. Marechal, E. Quesnel, and Y. Pauleau: Silver thin films deposited by magnetron sputtering. Thin Solid Films 241, 34 (1994).

20. B.D. McKay and A. Piperno: Practical graph isomorphism, II. $J$. Symb. Comput. 60, 94 (2014).

21. L.J. Vernon: Modelling growth of rutile $\mathrm{TiO}_{2}$. Ph.D. thesis, Loughborough University, Loughborough U.K., 2010.

22. G.T. Barkema and N. Mousseau: Event-based relaxation of continuous disordered systems. Phys. Rev. Lett. 77, 4358 (1996).

23. G. Henkelman and H. Jónsson: A dimer method for finding saddle points on high dimensional potential surfaces using only first derivatives. J. Chem. Phys. 111, 7010 (1999).

24. L. Bai and D. Breen: Calculating center of mass in an unbounded 2D environment. J. Graph. Tools 13, 53 (2008).

25. G. Henkelman and H. Jónsson: Improved tangent estimate in the nudged elastic band method for finding minimum energy paths and saddle points. J. Chem. Phys. 113, 9978 (2000).

26. F. El-Mellouhi, N. Mousseau, and L.J. Lewis: Kinetic activationrelaxation technique: An off-lattice self-learning kinetic Monte Carlo algorithm. Phys. Rev. B 78, 153202 (2008).

27. T. Lazauskas, S.D. Kenny, and R. Smith: Influence of the prefactor to defect motion in $\alpha$-iron during long time scale simulations. J. Phys.: Condens. Matter 26, 395007 (2014).

28. B.P. Uberuaga, M. Leskovar, A.P. Smith, H. Jónsson, and M. Olmstead: Diffusion of Ge below $\mathrm{Si}(100)$ surface: Theory and experiment. Phys. Rev. Lett. 84, 2441 (2000).

29. M. Chugh and M. Ranganathan: Kinetic Monte Carlo simulations of epitaxial growth of wurtzite $\mathrm{GaN}(0001)$. Phys. Status Solidi $C$ 12, 408 (2015).

30. G. Hu, G. Orkoulas, and P.D. Christofides: Regulation of film thickness, surface roughness and porosity in thin film growth using deposition rate. Chem. Eng. Sci. 64, 3903 (2009).

31. M.A. Novotny: Monte Carlo algorithms with absorbing Markov chains: Fast local algorithms for slow dynamics. Phys. Rev. Lett. 74, 1 (1995).

32. A.L. Lloyd, R. Smith, and S.D. Kenny: Critical island size for Ag thin film growth on $\mathrm{ZnO}(000-1)$. Nucl. Instrum. Methods Phys. Res., Sect. B 393, 22 (2017). 